SHORT REPORT

\title{
Automatic external defibrillation in a 6 year old
}

\author{
B König, J Benger, L Goldsworthy
}

Arch Dis Child 2005;90:310-311. doi: 10.1136/adc.2004.054981

A case is reported in which an automatic external defibrillator (AED) was used during the successful resuscitation of a 6 year old child in out-of-hospital cardiac arrest, despite the fact that these devices are not recommended in children under 8 years. The interpretation of resuscitation protocols is discussed and new developments in this area reported.

W idespread introduction of automatic external defibrillators (AEDs) has resulted in improved outcome from ventricular fibrillation (VF). ${ }^{1}$ These devices are designed to be used by people with little or no training, and their placement in strategic public areas (such as airports and casinos) has been reported favourably. ${ }^{2}$ AEDs are now commonly used in pre-hospital care.

In children, the recommended first shock for VF is $2 \mathrm{~J} / \mathrm{kg}$ using a monophasic defibrillator, ${ }^{3}$ but the data supporting this are scant. ${ }^{4}$ AEDs are designed for use in adults, and commonly deliver between $150 \mathrm{~J}$ and $200 \mathrm{~J}$ using a biphasic waveform. In younger children this could exceed the $10 \mathrm{~J} / \mathrm{kg}$ that is thought to be potentially damaging to the myocardium, and as a result AEDs are not recommended in children under 8 years. ${ }^{5}$ Although ventricular fibrillation is less frequent in children than in adults, deciding whether or not to use an available AED on any child under 8 has been described as a "terrible dilemma", 6 particularly in a protocol driven clinical environment.

\section{CASE REPORT}

Our local ambulance despatch centre received an emergency call regarding a 6 year old girl (weight $20.4 \mathrm{~kg}$ ), who had collapsed and appeared to be in cardiac arrest. Basic life support was initiated by the girl's mother according to instructions given over the telephone. The first paramedic, a "rapid response unit", arrived within 4 minutes. The child was apnoeic and pulseless. A Heartstream FR2 (Agilent Technologies, Palo Alto, CA, USA) automatic external biphasic defibrillator was attached to the child's chest and the monitor showed asystole. Cardiopulmonary resuscitation (CPR) was commenced, and the child ventilated with oxygen. After one minute a rhythm change to VF was noted and the AED analysed this as a shockable rhythm. One shock of

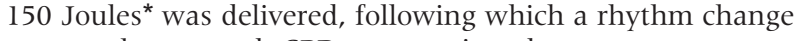
to asystole occurred. CPR was continued.

On the arrival of paramedic colleagues circulatory access was gained, the first dose of adrenaline was given, three cycles of the asystole protocol were completed, and endotracheal intubation was performed. After the third dose of adrenaline a rhythm change to VF was noted. A second shock of $150 \mathrm{~J}$ was delivered via the $\mathrm{AED}$, again followed by asystole. One minute later a

* The Agilent Heartstream FR2 AED is a biphasic defibrillator that adjusts the waveform and delivered charge according to the measured chest impedance, but the delivered energy can only vary from 140 to 161 Joules. narrow complex bradycardia emerged with a palpable carotid pulse. The heart rate gradually increased and spontaneous respiratory effort occurred.

On arrival at the hospital the child had a palpable pulse of 80 beats/min, a respiratory rate of six, and reactive pupils. Assisted ventilation was continued. A 12 lead electrocardiogram showed a prolonged QT interval. She was initially admitted to our paediatric intensive care unit. Computed tomography of her brain and an electroencephalogram were unremarkable. Six days after her cardiac arrest the patient was conversing and self-caring. An implantable defibrillator was inserted on the assumption that her arrest was secondary to prolonged QT interval causing ventricular fibrillation. She has subsequently returned to school.

\section{DISCUSSION}

This is the second recorded case of successful resuscitation following defibrillation with an AED in a child younger than 8 years. Neither the previously reported 3 year old, ${ }^{7}$ nor our 6 year old child, had evidence of any myocardial damage, though in our case this is supported by echocardiographic evidence rather than additional cardiac enzyme analysis.

The existing monophasic $2 \mathrm{~J} / \mathrm{kg}$ recommendation was deduced from a study of 71 transthoracic defibrillations performed on 27 children. ${ }^{4}$ Subsequent clinical experience suggests that this dose is effective, but the optimal dose is unknown. ${ }^{4}$ In addition, evidence is emerging to suggest that damage to the myocardium may be less with modern biphasic rather than traditional monophasic waveforms. ${ }^{8}$

Defibrillation is employed to depolarise the fibrillating heart, causing a temporary halt to electrical activity and providing an opportunity for a cardiac rhythm to be reestablished. Use of higher energies may cause a prolonged depolarisation (asystole), and this may explain why the second shock was initially followed by apparent asystole before the emergence of a perfusing rhythm.

Current research in adult and animal defibrillation explores the analysis of both the defibrillation waveform and the transthoracic impedance, potentially enabling a shock with optimal energy, timing, and waveform to be delivered. ${ }^{9}$ Application of these principles is leading to development of paediatric defibrillators using lower energies. In the current absence of such equipment, however, professionals facing VF arrest in young children with an "adult" AED to hand will continue to face a "dilemma".

Most practitioners are now familiar with the initial part of current recommendations: "set voltage AEDs should not be used in children under the age of 8 years". Until new "child friendly" AEDs become available, we would use this case to draw attention to the additional statement that: "withholding use of an AED if no other defibrillator is available could cause harm"'. ${ }^{10}$

\section{ACKNOWLEDGEMENTS}

The parents of the child, and the staff of the Children's Emergency Department, take this opportunity to wholeheartedly praise the skills and the professionalism of the ambulance personnel, who we believe saved this girl's life. 


\section{Authors' affiliations}

B König, L Goldsworthy, Department of Paediatric Emergency

Medicine, Bristol Royal Hospital for Children, UK

J Benger, Department of Emergency Medicine, Bristol Royal Infirmary, UK

Competing interests: none declared

Correspondence to: Dr J Benger, Paediatric Emergency Department. Bristol Royal Hospital For Children, Paul O'Gorman Building, Upper Maudlin Street, Bristol BS2 8BJ, UK; jonathan.benger@ubht.swest.nhs.uk

Accepted 17 September 2004

\section{REFERENCES}

1 Marenco JP, Wang PJ, Link MS, et al. Improving survival from sudden cardiac arrest: the role of the automated external defibrillator. JAMA $2001 ; 285: 1193-2000$.
2 Liddle R, Davies CS, Colquhoun $M$, et al. $A B C$ of resuscitation: the automated external defibrillator. BMJ 2003;327:1216-18.

3 Molyneux EM, Mackway-Jones K (Advanced Life Support Group). Advanced paediatric life support: the practical approach, 3rd edn. London: BMJ Books, 2001.

4 Gutgesell HP, Tacker WA, Geddes LA, et al. Energy dose for ventricular defibrillation of children. Pediatrics 1976;58:898-901.

5 Resuscitation Council (UK) Guidelines, 2001. Available from: http:// www.resus.org.uk/pages/aed.htm\#child (accessed 31 December 2003).

6 Senior K. Automatic external defibrillation is safe in small children. Lancet 2001;357:1678.

7 Gurnett CA, Atkins DL. Successful use of a biphasic waveform automated external defibrillator in a high-risk child. Am J Cardiol 2000;86:1051-3.

8 Tang W, Weil MH, Sun S, et al. The effects of biphasic and conventional monophasic defibrillation on post resuscitation myocardial function. J Am Coll Cardiol 1999;34:815-22.

9 Niemann JT. Defibrillation waveforms. Ann Emerg Med 2001;37:59-61.

10 Biarent D, Coovadia A, Hazinski MF, et al. Use of automated external defibrillators for children: an update. An advisory statement from the Pediatric Advanced Life Support Task Force, International Liaison Committee on Resuscitation. Resuscitation 2003;57:237-43.

\section{Long term effects of sodium valproate in pregnancy}

$\square$ he management of women with epilepsy may involve difficult choices during pregnancies. Inadequate seizure control during pregnancy may harm both mother and foetus but antiepileptic drugs may also harm the foetus. A study in Liverpool and Manchester ( $\mathrm{N}$ Adab and colleagues Journal of Neurology, Neurosurgery, and Psychiatry 2004;75:1575-83) has pointed to treatment with sodium valproate during pregnancy as a particular risk factor for developmental and cognitive problems in the child.

A total of 547 mothers were identified through epilepsy and antenatal clinics, and 219 $(40 \%)$ agreed to take part in the study. The mothers were interviewed and their 375 children aged 6 months to 16 years were examined. Two hundred and forty nine of 256 children aged over 5 years had WISC IQ tests. Among these 249, 80 had not been exposed in utero to antiepileptic drugs, 120 had been exposed to monotherapy (41 with valproate, 52 with carbamazepine, 21 with phenytoin, and six with other drugs), and 49 had been exposed to polytherapy (28 including valproate). Full scale and performance IQs did not vary significantly according to drug exposure in utero. Verbal IQ scores, however, were significantly lower (by 7 points on average) in children exposed to valproate monotherapy compared with children exposed to carbamazepine, phenytoin monotherapy, or unexposed. Among children whose mothers took valproate monotherapy in pregnancy $42 \%$ had a verbal IQ score of less than $80(22 \%,<70)$. Exposure to valproate monotherapy in utero was associated with a 3.5 fold increase in the risk of a verbal IQ under 70 compared with no exposure to antiepileptic drugs. Three factors (low maternal IQ, valproate exposure, and frequent tonic-clonic seizures $(>4)$ in pregnancy) were independently predictive of low verbal IQ in children aged 6 or more years. Children exposed to valproate were more likely to have additional educational needs. The authors of this paper call for an increase in the provision of information and counselling about anti-epileptic drug treatment during pregnancy for all women with epilepsy in the child bearing age group before they become pregnant. There was a significant correlation between increasing dose of valproate in the first trimester and lower verbal IQ with no significant effect at doses of less than $800 \mathrm{mg}$ a day. Among children under the age of 6 years exposure to valproate in utero was associated with delays in the interaction and hearing and language domains of the Schedule of Growing Skills II test. The prevalence of moderate or severe dysmorphic features was $44 \%$ (valproate), 9\% (carbamazepine), and 2\% (unexposed), and there was an association between dysmorphic features and low verbal IQ. 